Methods A prospective analysis of all Micra VA implants performed at Royal Papworth Hospital was carried out. This included pacing checks for up to 24 months post device insertion, evaluating complications and specifically noting thresholds and R-wave amplitude changes.

Results A total of 24 Micra leadless pacemakers were implanted at our centre between 2017 and 2020. The age range for the patients was 37 to 92 years, mean age $71 \pm$ 13 yrs. 8 out of 24 (33\%) patients had poor venous access, with bilateral subclavian obstruction, requiring the use of a leadless pacemaker. 2 out of 24 had bilateral previous pacemaker infections and extraction. For the remaining patients, $12 / 24(50 \%)$ had atrial fibrillation with slow ventricular response as the primary indication for the device. 6 out of $24(25 \%)$ patients had a history of LV impairment (4 patients severe LVSD, 2 moderate LVSD). One patient had a previous cardiac transplant.The implant was successful for all patients. One patient required the procedure to be repeated under general anaesthetic as she did not tolerate the insertion of the femoral sheath under sedation. 2 patients $(8 \%)$ required repositioning of the device during the case, as initial placement was unsatisfactory. 23/24 patients had the device placed in the septum and 1 patient in the RV apex. This patient had undergone multiple previous tricuspid valve surgeries, which made septal positioning challenging, and so an apical placement was accepted. Mean procedure time was $62 \pm 16$ mins. Mean fluoroscopy time was $5.8 \pm 4.2$ mins. Implant threshold was $0.6 \mathrm{~V} \pm$ $0.4 \mathrm{~V}$. Threshold at 1 year follow up was $0.5 \mathrm{~V} \pm 0.2 \mathrm{~V}$ and $0.6 \mathrm{~V} \pm 0.2 \mathrm{~V}$ at 2 years. Paired $\mathrm{T}$ testing showed no statistically significant difference in threshold values at implant, and year $1(\mathrm{p}=0.7, \mathrm{n}=13)$, or implant and year $2(\mathrm{p}=0.78, \mathrm{n}=6)$. The $\mathrm{R}$ wave at implant was $9.5 \pm$ $4.1 \mathrm{mV}$, and $9.8 \mathrm{mV} \pm 2.5 \mathrm{mV}$ at year 1 , again with no statistically significant difference $(\mathrm{p}=0.87, \mathrm{n}=13)$. Mean battery life at 2 year follow up was $7 \pm 0.5$ years. Pacing percentages varied from $0.1 \%$ to $99.99 \%$.

Conclusion Although are numbers are small, particularly for follow up over 2 years $(n=6)$, the initial results are encouraging, and support a low complication rate, and no evidence of premature battery failure or issues with device threshold requiring re intervention. It is imperative that further studies are carried out to give a picture of longer term follow up for the leadless Micra VA pacemaker focussing on these two key issues.

Conflict of Interest nil

\section{IMPLANTABLE CARDIOVERTER-DEFIBRILLATORS IN THE ELDERLY: A SINGLE-CENTRE OBSERVATIONAL STUDY ON OUTCOMES IN OCTOGENARIANS}

${ }^{1}$ Shermayne Ng, ${ }^{1}$ Jamie Kay*, ${ }^{1}$ Manoj Makharia, ${ }^{1}$ Caroline Wick, ${ }^{2}$ Raj Khiani. 'Barnet General Hospital, London, UK; ${ }^{2}$ Royal Free London NHS Foundation Trust

10.1136/heartjnl-2021-BCS.105

Introduction Implantable cardioverter-defibrillators (ICDs) are well-established therapy for sudden cardiac death (SCD) prevention. However, the average age of patients in both primary and secondary prevention clinical trials of ICDs has been in the 60 s, with less than $25 \%$ of included participants above the age of 75 . As such, data supporting the clinical and costeffectiveness of ICDs in this understudied age group is lacking. We aim to review the outcome of patients $>80$ years of age with ICD therapy in a district general hospital serving an elderly population.

Methods Patients > 80 years of age who underwent ICD implantation between 2015 and 2017 were identified from the hospital electronic records. Conventional ICD and cardiac resynchronisation therapy with defibrillator (CRT-D) implants were included. Primary outcomes include overall all-cause mortality at time of data collection and at 1-year following implant. Secondary outcomes include number of patients receiving appropriate and inappropriate therapy and complication rates.

Results We identified 38 patients $>80$ years of age who underwent a defibrillator implantation in this period. 17 and 21 patients received an ICD and CRT-D respectively. 29 $(76 \%)$ were male and the mean age at implant is 83.3 years. The mean follow-up period was 37.3 months (range: 6 - 51 months). The average number of co-morbidities per patient was 4 . The most common comorbidities were heart failure with reduced ejection fraction (32/38), ischaemic heart disease (27/38), hypertension (22/38) and atrial fibrillation (21/ 38). 11 of 38 patients had chronic kidney disease. 25 patients $(66 \%)$ underwent ICD implantation as primary prevention therapy. The overall mortality rate was $26.3 \%$ and the 1 -year mortality rate was $2.6 \%$. The average time to death from implant was 2.2 years. 6 patients (16\%) received appropriate shocks during the follow-up period. No patients received inappropriate shocks. There were no acute or late complications from device implantation in the follow-up period.

Conclusion In this single-centre observational study, ICD implantation in octogenarians had a low complication rate. The majority of patients survived beyond 12 months, although the average time from implant to death was just over 2 years. ICD therapy may be beneficial in octogenarians and patients should be considered for ICD therapy after careful selection and counselling of risks and benefits. Further work is required to identify patients in this age group whom may benefit the most from ICD therapy.

Conflict of Interest None

\section{A MIXED-REALITY HOLOGRAPHIC VIEWING PLATFORM ENABLING INTERACTION WITH 3D ELECTROANATOMICAL MAPS USING THE HOLOLENS}

${ }^{1}$ Anura Malaweera, ${ }^{2}$ Ramit Jogi, ${ }^{1}$ Matthew Wright, 'Mark O'Neill, 'Steven Williams. ${ }^{1}$ St. Thomas Hospital, London, UK; ${ }^{2}$ Kings College London

\subsection{6/heartjnl-2021-BCS.106}

Introduction Three dimensional (3D) electroanatomical maps (EAMs) created during electrophysiology procedures are traditionally displayed on 2D monitors connected to mapping systems. This has limitations, such as the lack of interaction with EAMs, the need for another user to control them, and the size of EAM displayed, which is limited by the resolution of these monitors. To overcome these, we created a novel technology to display EAMs on a mixed reality (MR) platform.

Methods We used the Microsoft ${ }^{\circledR}$ HoloLens to create this MR platform. Studies from patients who had already undergone catheter ablation for atrial fibrillation, where EAMs of 